\title{
Blurring the Germline: Genome Editing and Transgenerational Epigenetic Inheritance.
}

Tim Lewens

University of Cambridge

Department of History and Philosophy of Science

Free School Lane

Cambridge CB2 3RH

Email: tml1000@cam.ac.uk

\section{Abstract}

Sperm, eggs and embryos are made up of more than genes, and there are indications that changes to non-genetic structures in these elements of the germline can also be inherited. It is, therefore, a mistake to treat phrases like 'germline inheritance' and 'genetic inheritance' as simple synonyms, and bioethical discussion should expand its focus beyond alterations to the genome when considering the ethics of germline modification. Moreover, additional research on non-genetic inheritance draws attention to a variety of means whereby differences can be inherited in offspring generations that do not rely on differences in germline structures. Research on these diverse forms of inheritance challenges the notion that there is some special form of ethical concern that falls on germline interventions in general, and on interventions to the nuclear genome within the germline in particular. 


\section{Keywords}

Epigenome Editing, Genome Editing, Germ Cells, Germline Inheritance, HFEA, Mitochondrial Donation, Transgenerational Epigenetic Inheritance.

\section{EPIGENETICS AND THE GERMLINE}

Bioethicists are beginning to pay attention to research on forms of non-genetic inheritance, and especially to epigenetic inheritance. In particular, they have pointed to ways in which traditional public health concerns may need to address the impacts of a variety of nutritional and environmental factors on the health of multiple generations. ${ }^{1}$ This article addresses a related question. Do we need to rethink our understanding of the ethical significance of germline interventions, in the light of work that draws attention to a variety of non-genetic inheritance mechanisms? ${ }^{2}$

${ }^{1}$ Loi, M., L. Del Savio \& E. Stupka. Social Epigenetics and Equality of Opportunity. Public Health Ethics 2013; 6: 142-153; Juengst, E., J. Fishman, M. McGowan \& R. Settersten. Serving Epigenetics Before its Time. Trends Genet 2014; 30: 427-429; Dupras, C., V. Ravitsky \& B. Williams-Jones. Epigenetics and the Environment in Bioethics. Bioethics 2014; 28: 327-334.; Del Savio, L., M. Loi and E. Stupka. Epigenetics and Future Generations. Bioethics 2015; 29: 580-587.

${ }^{2} \mathrm{~A}$ valuable taxonomy of these many different forms of inheritance is given by Jablonka, E. \& M. Lamb. 2014. Evolution in Four Dimensions. Revised Edition. Cambridge, MA: MIT Press. 
If we want to understand why differences between organisms of one generation are reflected in similar differences between their offspring, we must look beyond differences in transmitted DNA. In a recent review, Miska and Ferguson-Smith opened with the claim that, 'It is now clear that inheritance not based on DNA sequence exists in multiple organisms, with examples found in microbes, plants, and invertebrate and vertebrate animals'. ${ }^{3}$ In spite of the caution they urged with respect to our knowledge of the mechanisms and significance of non-genetic inheritance in mammals, they indicated that these forms of inheritance, "have major implications for human health'. ${ }^{4}$

The catch-all term 'non-genetic inheritance' encompasses all processes by which differences between adults reliably re-appear in their offspring in ways that are not explained by differences in transmitted genes. ${ }^{5}$ One such form of inheritance occurs

\footnotetext{
${ }^{3}$ Miska, E. \& A. Ferguson-Smith. Transgenerational Inheritance: Models and Mechanisms of non-DNA Sequence-based Inheritance. Science 2016; 354: 59. 4 Ibid: 63.
}

${ }^{5}$ This general way of describing inheritance processes is neutral between those who think in terms of a series of distinct inheritance mechanisms, processes or channels, and those (often influenced by developmental systems theory) who are sceptical of 'channel talk'. For various suggestions for how to resolve these debates see Griffiths, P. \& R. Gray. Developmental Systems and Evolutionary Explanation J Philos 1994; 91: 277-304; Mameli, M. The Inheritance of Features Biol Philos 2005; 20: 365-399; 
when the inheritance of difference is explained via differences in non-genetic structures within sperm and egg cells. These are consequently classed by biologists as instances of non-genetic germline inheritance. Bohacek and Mansuy have claimed that, 'Evidence for germline-dependent non-genetic inheritance of acquired traits in mammals has accumulated in neuroscience, behavioural neuroendocrinology, environmental toxicology and nutritional science'. ${ }^{6}$ The work they review draws attention to the possibility that changes to organisms that occur across their lives can be passed onto offspring via changes to gametes, yet not via changes to DNA sequence. ${ }^{7}$ This presents bioethicists with a question: what does research on non-genetic inheritance-including non-genetic germline inheritanceimply for the ethical disapproval usually reserved for germline genetic modification?

Lewens, T. 2015. Cultural Evolution: Conceptual Challenges. Oxford: Oxford University Press; Lewens, T. Human Nature, Human Culture: The Case of Cultural Evolution Interface Focus 2017; 7: 20170

${ }^{6}$ Bohacek, J. \& I. Mansuy. Molecular Insights into Transgenerational Non-Genetic Inheritance of Acquired Behaviours. Nat Rev Genet 2015; 16: 641.

${ }^{7}$ Roy et al have noted a potential implication of this research: the use of assisted reproductive technologies may provoke epigenetic risks for developing embryos, which are passed to future generations. See Roy, M-C, C. Dupras \& V. Ravitsky. The Epigenetic Effects of Assisted Reproductive Technologies: Ethical Considerations. J Dev Orig Health Dis 2017; 8: 436. 
This article proceeds as follows. Section two asks what scientists mean by 'the germline'. It argues that the notion is understood by reference to lineages of germ cells, and that it is a mistake to equate 'the germline' with the transmitted genetic material that forms just one part of those cells. Section three briefly surveys research indicating the importance of various different forms of non-genetic inheritance. Section four displays the consequent tension between a comparatively narrow understanding of 'the germline' in bioethical circles, which typically presupposes that phrases like 'germline inheritance' and 'genetic inheritance' are simple synonyms, and a broader understanding of 'the germline' in scientific circles, where forms of non-genetic germline inheritance are widely recognised. This section also notes some potential regulatory implications-for example, for the wording of the HFEA Act (2008) - of the possibility of inheritable interventions to structures other than DNA. ${ }^{8}$ In short, the first half of the article establishes that bioethicists have often assumed without argument that 'germline inheritance' and 'genetic inheritance' are synonymous.

\footnotetext{
${ }^{8}$ Human Fertilisation and Embryology Act (2008) https://www.legislation.gov.uk/ukpga/2008/22/pdfs/ukpga 20080022 en.pdf
} Accessed 15th March 2018. 
The second half of this article explores the significance of correcting bioethicists' presuppositions about mechanisms of inheritance. ${ }^{9}$ This is a challenging topic, because the overall empirical picture coming from research on trans-generational non-genetic inheritance remains unclear. Section five assesses the reasons given for subjecting germline genetic interventions to ethical scrutiny, in order to determine whether there are salient ethical features that uniquely apply to these ways of influencing inheritance. It sketches a case for thinking there are no such salient differences. Sections six and seven then consider, and reject, two potential responses to this effort to blur the ethical significance of germline genetic interventions; one is based on the alleged impact of genomic changes on 'identity', the other on the irreversibility of genomic alterations.

\section{THE GERMLINE IN SCIENTIFIC WORK}

What is meant by 'the germline'? Frankel \& Hagen state that the term, 'refers to genetic material that is hereditable from parent to child'. ${ }^{10}$ The Oxford English

${ }^{9}$ Early reflections consistent with this paper's argument can be found in Robertson, J. Oocyte Cytoplasm Transfers and the Ethics of Germ-Line Intervention. J Law Med Ethics 1998; 26: 217.

${ }^{10}$ Frankel, M. \& B. Hagen. 2011. Background Paper: Germline Therapies. http://nuffieldbioethics.org/wp- 
Dictionary defines the 'germ line' as 'a series of germ cells each descended from earlier cells in the series, regarded as continuing through successive generations of an organism'. ${ }^{11}$ The OED definition of 'germ line' is therefore more liberal than Frankel \& Hagen's, because germ cells-like all cells-contain far more than just genetic material.

In the face of such disagreement, we can follow Isasi et al in requesting 'scientific understanding and precision in legal definitions of what constitutes an embryo and/or its germ line'..$^{2}$ In scientific contexts, the lengthiest efforts at explicit definition and description tend to concern germ cells. According to developmental biologists, the function of germ cells is reproductive. Moreover, they transmit more than DNA to future generations. They, 'are a central component of sexual reproduction in animals. They are the route by which the genome and cytoplasmic components are

content/uploads/Germline therapies background paper.pdf Accessed 15th March

2018.

11 OED online. germ, n. 2018. Oxford University Press.

http://www.oed.com/view/Entry/77860?redirectedFrom=germline (accessed March $15,2018)$.

${ }^{12}$ Isasi R., E. Kleiderman \& B. M. Knoppers. Editing policy to fit the genome?

Science 2016; 351: 337-9. 
transferred to the next generation'.13 These domains of biology are full of detailed descriptions of when and how germ cells arise as the early embryo develops. ${ }^{14}$

These biologists do not usually think of embryos and gametes as germ cells. Instead, germ cells - 'the precursors of sperm and eggs'-emerge later in development..$^{15}$ They do, however, describe embryos and gametes as elements of the germline: 'life experiences can induce epigenetic changes in the germline (sperm and eggs)'. ${ }^{16}$ The germline is not equated here with an uninterrupted sequence of

${ }^{13}$ Schedl, T. Germ Cell. Encylopedia of Genetics. Academic Press 2001: 837; emphasis added.

${ }^{14}$ The following is typical: 'In the mouse, the germ cells, once they have formed, migrate through the tissues of the embryo to the gonad primordia... where they coassemble with somatic gonadal cells to form the sex cords'; Wylie, C. \& R. Anderson 2002. Germ Cells. In Mouse Development. Rossant and Tam, eds. Elsevier. Surani et al claim that 'Germ cells are highly specialized cells established by a specific transcriptional program that includes repression of the somatic fate': Surani, A., K. Hayashi \& P. Hajkova. Genetic and Epigenetic Regulators of Pluripotency. Cell 2007; 128: 747.

15 Surani, A. 2018. Plain English: Germ cells.

https://www.gurdon.cam.ac.uk/research/surani Accessed 15th March 2018 ${ }^{16}$ Gapp, K. \& J. Bohacek. Epigenetic Germline Inheritance in Mammals: Looking to the Past to Understand the Future. Genes, Brain Behav 2017 doi: 10.1111/gbb.12407: 1. 
germ cells that descends down generations. The fact that, in mammals, the appearance of germ cells is a result of developmental differentiation means there is no such uninterrupted sequence. ${ }^{17}$ Even so, there is a link between the concept of the germline and the concept of a germ cell. An inherited germline interventionwhether it is wrought directly on germ cells, or on sperm, eggs or the cells of early embryos - is one that is passed across generations via corresponding changes to germ cells sensu stricto.

\section{NON-GENETIC GERMLINE INTERVENTIONS}

There is disagreement among scientists regarding the nature of epigenetic inheritance. There is also a widespread sense that its significance is exaggerated in communications to non-specialist audiences. ${ }^{18}$ Miska and Ferguson-Smith, for example, note that while there is a clear case for non-genetic inheritance in many plant and animal organisms, 'In mammals, the molecular mechanisms have been

17 Johnson, M. The Developmental Basis of Identity. Stud Hist Philos Biol Biomed Sci 2001; 32: 601-617.

18 Grossniklaus, U., W. Kelly, A. Ferguson-Smith, M. Pembrey \& S. Lindquist. Transgenerational Epigenetic Inheritance: How Important is it? Nat Rev Genet 2013; 14: 228-235; Meloni, M. Heredity 2.0: The Epigenetics Effect. New Genet Soc 2015; 34: 117-124; Meloni, M. and G. Testa. Scrutinizing the Epigenetics Revolution Biosocieties 2014; 9: 431-456. 
challenging to elucidate'. ${ }^{19}$ They are confident of a variety of phenomena in humans whereby alterations to epigenetic states result in the inheritance of phenotypes over a small number of generations. They believe there is not, as yet, enough evidence to explain how this occurs.

Others are more confident of the existence of important forms of epigenetic inheritance in mammals, and of the mechanisms that explain it. Gapp \& Bohacek have argued that: 20

While [the] concept of epigenetic germline inheritance [in mammals] has long been met with skepticism, evidence in support of this route of information transfer is now overwhelming, and some key mechanisms underlying germline transmission of acquired information are emerging.

When considering the nature of non-genetic inheritance, and the apparent disputes over its significance that characterise the remarks quoted above, it is useful to bear in mind a threefold distinction between different types of non-genetic inheritance

${ }^{19}$ Miska \& Ferguson-Smith, op. cit. note 3, p. 59; for further sceptical assessments see also Heard, E. \& R. Martienssen. Transgenerational Epigenetic Inheritance: Myths and Mechanisms. Cell 2014; 157: 95-109; Kazachenka et al. Identification, Characterization, and Heritability of Murine Metastable Epialleles: Implications for Non-Genetic Inheritance. Cell 2018; 175: 1259-1271.

${ }^{20}$ Gapp \& Bohacek, op. cit. note 16, p.1. 
process. ${ }^{21}$ Two involve the inheritance of difference mediated by differences in germ cells, the third does not implicate germ cells in this way. We begin with the third.

\section{Non-Germ-Cell-based Inheritance}

It is possible for parents to affect their offspring in ways that result in the reconstruction of resembling traits in the new generation, even when this is not effected via differences in the germ cells they transmit. For example, Bohacek \& Mansuy suggest that, 'The composition of the seminal fluid [rather than the gametes contained within it], which is transferred to the female with sperm during mating, can change with the environment and influence the offspring independently of sperm'. ${ }^{22}$ Whitelaw \& Whitelaw give a different example in rats: 'The offspring of greying mothers exhibit greying but the offspring of greying fathers do not....[An] elegant study involving caesarean sections and foster nursing showed that it was the result of transmission from the mother to newborns of a murine leukaemia virus (probably via the milk) that causes greying'. ${ }^{23}$ In both cases, when differences are inherited across generations it is not because of differences in what is passed within gametes.

Germline Epigenetic Inheritance

\footnotetext{
${ }^{21}$ Miska \& Ferguson-Smith, op. cit. note 3.

22 Op. cit. note 3.

${ }^{23}$ Whitelaw, N. \& E. Whitelaw. Transgenerational Epigenetic Inheritance in Health and Disease. Curr Opin Genet Dev 2008; 18: 276.
} 
Biologists have distinguished two ways in which alterations to non-DNA-sequence structures within germ cells may turn out to influence inheritance: transgenerational epigenetic inheritance, and intergenerational epigenetic inheritance. ${ }^{24}$

Transgenerational epigenetic inheritance involves changes to germline epigenetic structures persisting over multiple reproducing generations, in a way that continues beyond the event that initiated an epigenetic alteration. This would potentially give epigenetic systems of inheritance a number of similar properties -in terms of fidelity and longevity - to the genetic inheritance system. Intergenerational epigenetic inheritance instead occurs when an environmental effect on (for example) a pregnant female directly affects not only the phenotype of the maturing embryo within the parent, but it also has an influence on the germ cells within that very embryo; that is, it also affects what the embryo will bequeath to its own progeny. The result is that an effect can potentially be observed in three generations - parent, offspring and grandoffspring-even if there is no tendency for the epigenetic alterations in question to be preserved faithfully across further reproductive cycles. This is why an important test for true transgenerational inheritance, as opposed to intergenerational inheritance, is to ask whether epigenetic modifications persist into a fourth generation following an environmental insult to the pregnant organism.

There is debate over the extent to which transgenerational epigenetic inheritance has been demonstrated in mammals, although the effect seems well confirmed over

${ }^{24}$ Miska \& Ferguson-Smith, op. cit. note 3. 
many generations in plants. Scientists have tended to assume, for example, that epigenetic marks are 'reset' or 'reprogrammed' with the establishment of each new mammalian generation, with the result that in the case of methylation, differences are not inherited across generations. ${ }^{25}$ However, there are indications that this resetting may not apply to all areas of the genome. Hackett et al, for example, noted that in mice there are 'rare regulatory elements that escape systematic DNA demethylation in PGCs [primordial germ cells], providing a potential mechanistic basis for transgenerational epigenetic inheritance'. ${ }^{26}$

There are also problems in establishing with confidence that alterations to epigenetic marks, rather than alterations in DNA sequence, ground the explanation of transgenerational inheritance. ${ }^{27}$ One problem that Ferguson-Smith draws our attention to is, "the fact that DNA methylation is a mutagen that contributes to $\mathrm{C}$ to $\mathrm{T}$ transitions if

25 Heard \& Martiennssen, op. cit. note 19.

${ }^{26}$ Hackett, J. et al. Germline DNA Demethylation Dynamics and Imprint Erasure Through 5-Hydromethylcytosine. Science 2013; 339: 448-452.

${ }^{27}$ Some commentators have raised problems for the very distinction between epigenetic and genetic systems: see Dupras, C. V., L. Song, K. Saulnier \& Y. Joly Epigenetic Discrimination: Emerging Applications of Epigenetics Pointing to the Limitations of Policies Against Genetic Discrimination. Front Genet 2018; 9: 202; Lappé M., Landecker H. How the genome got a life span. New Genet Soc 2015; 34: 152-176. 
not repaired'. ${ }^{28}$ In other words, an environmental intervention that causes an alteration in an epigenetic methylation state might have, as a knock-on effect, a further alteration in DNA sequence which could then persist over several generations. The upshot is that it is hard to tell, even if we observe a sequence of events that begins with an environmental change causing an epigenetic alteration, and which results in phenotypic changes that are transgenerationally stable, that we are dealing with non-genetic inheritance.

\section{THE GERMLINE IN BIOETHICS}

The question of how important transgenerational epigenetic inheritance is for our own species, and for other mammalian species, is unclear. Yet the preceding sections have established that there is an important question to be asked about whether such transgenerational epigenetic inheritance is real, hence whether there are potential ways of affecting the germline, with effects across multiple generations, that do not require alterations to DNA.

This speaks in favour of greater precision when reports and statements are issued condemning germline interventions. ${ }^{29}$ For example, the US National Academies'

28 Grossniklaus et al, op. cit. note 18, p. 233.

29 See Bonnickson, A. The Politics of Germline Therapy. Nat Genet 1998; 19: 10-11; NCOB. 2016. Genome Editing: An Ethical Review. London: Nuffield Council on 
report on what they called 'mitochondrial replacement technologies' equates 'germline modification' with 'human inheritable genetic modification'. ${ }^{30}$ The American Association for the Advancement of Science's discussion of 'Human Inheritable Genetic Modifications' begins by avowing concern with the 'the scientific, ethical, religious, and policy issues associated with interventions in the human germ line.' Yet this comparatively broad brief quickly narrows to an assessment of 'the scientific prospects for inducing controlled inheritable genetic changes in human beings'. ${ }^{31}$ In both cases there is a misleading presupposition that 'interventions in the human germ line', and 'inheritable genetic changes' are simple synonyms.

Our discussion shows how major position statements on germline interventions often vary, apparently unwittingly, in their scope. First, there is a very general notion of what the UNESCO IBC refers to as interventions that might result in 'modifications

Bioethics; Scott, R. \& S. Wilkinson Germline Genetic Modification and Identity: The Mitochondrial and Nuclear Genomes. Oxf J Leg Stud 2017; 37: 886-915.

${ }^{30}$ National Academies of Sciences, Engineering and Medicine. 2016. Mitochondrial Replacement Techniques: Ethical, Social, and Policy Considerations. Washington, DC: National Academies.

${ }^{31}$ Frankel, M. \& A. Chapman. 2000. Human Inheritable Genetic Modifications.

Prepared by the American Association for the Advancement of Science. https://www.aaas.org/sites/default/files/migrate/uploads/germline2.pdf Accessed 15th March 2018. 
for descendants'. ${ }^{32}$ This maximally inclusive notion covers any change that might be inherited across generations, regardless of the mechanism. Second, there is a narrower notion - mentioned by the AAAS among others-of achieving transgenerational change via alterations to the germline. Third, there is an even narrower notion-directly addressed by the US National Academies-of changing the germline via the specific method of inducing genetic changes.

As things stand, the protection of DNA sequence often has a paramount place in regulations controlling the germline. In the UK, for example, only what are called 'permitted eggs' and 'permitted sperm' may be used for reproductive purposes. The UK HFEA Act states that (among other things) a permitted egg is one: 'whose nuclear or mitochondrial DNA has not been altered'. ${ }^{33}$

The Act tells us that it is alteration of DNA - rather than other molecular structures within the egg - that makes that an egg impermissible for reproductive purposes. But alterations to inheritable structures within sperm and eggs other than DNA sequence might also be passed on to offspring. As things stand, modifications to such germline structures are not explicitly prohibited by legislation.

${ }^{32}$ IBC. 2015. Report of the IBC on Updating its Reflection on the Human Genome and Human Rights. UNESCO International Bioethics Committee: Paris.

${ }^{33}$ HFEA op. cit. note 8. The act gives a parallel definition for 'permitted sperm'. 
The need for greater awareness and precision with respect to regulation is made pressing by the advent of established techniques of 'epigenome editing'. ${ }^{34}$ They typically use modifications of the CRISPR/Cas9 approach to allow the 'editing' not of DNA sequence itself, but of the methylation patterns that affect gene action. Should we learn that epigenetic modifications can sometimes be inherited across human generations, then we will need to contemplate modifying legislation to cover these non-DNA-sequence-based forms of germline 'editing'.

\section{WHY DO WE CARE ABOUT THE GERMLINE?}

So far this article has established two claims. First, international bioethical discussion regarding the rights and wrongs of medical interventions in the processes of inheritance tends to focus overwhelmingly on alterations to genes. Indeed, these discussions tend to equate the notion of potentially problematic 'germline' interventions with interventions to DNA sequence. Second, we have also seen a growing body of work indicating that inheritance can perhaps be achieved through a variety of non-genetic mechanisms. Some involve the inheritance of difference mediated via differences in non-genetic germline structures. Some of these only persist for a small number of generations. Some scientists believe (but much of this work remains conjectural) that epigenetic inheritance can also have more lasting trans-generational stability. The question, then, is how, if at all, discussion within

${ }^{34}$ E.g. Lui, X. S. et al. Rescue of Fragile X Syndrome Neurons by DNA Methylation Editing of the FMR1 Gene. Cell 2018; 172: 979-992. 
bioethics should be broadened in the light of emerging information about these forms of non-genetic inheritance, including non-genetic germline inheritance.

There are three options for how we might respond to this question:

1) Retain an ethical focus on germline inheritance, and retain the focus on interventions to nuclear DNA sequence, by arguing that forms of non-genetic inheritance do not raise the same ethical concerns as interventions to nuclear DNA.

2) Retain an ethical focus on germline inheritance, but expand this focus to include non-genetic germline inheritance, by arguing that all forms of germline inheritance raise distinctive ethical concerns.

3) In the light of work suggesting a variety of different means-some which involve differences in germline structures, some which do not-by which inheritance across generations can be effected, argue that the concept of the germline has no distinctive ethical significance.

We cannot decide between these three options unless we examine why ethical concern has been expressed about germline interventions in the first place. Only then can we understand whether interventions to the nuclear genome are the only ones with the potential to trigger the intended form of ethical concern.

Ethical concern sometimes focuses on the germline simply because of the potential for changes to germ cells to persist over more than one generation. In other words, 
ethical focus is not on the germline per se, but rather on the prospect of changes that are passed to future generations regardless of how this is achieved. Consider again the UNESCO IBC's 'Update' Statement: 'In several countries somatic gene therapy has received ethical and regulatory acceptance because the genetic changes induced are not passed on to the next generation'. ${ }^{35}$ If we think the problem with germline interventions is simply that they have effects that are 'passed on to the next generation', then we potentially open up the whole range of putative inherited epigenetic modifications-both inter-generational and trans-generational-to precisely the same set of worries.

Sometimes, however, a richer set of concerns is raised. The NIH's statement on genome editing notes that:

...the strong arguments against engaging in this activity remain. These include the serious and unquantifiable safety issues, ethical issues presented by altering the germline in a way that affects the next generation without their consent, and a current lack of compelling medical applications justifying the use of CRISPR/Cas9 in embryos. ${ }^{36}$

35 IBC op. cit. note 32.

${ }^{36} \mathrm{NIH} .2015$. Statement on NIH Funding of Research Using Gene-Editing

Technologies in Human Embryos. https://www.nih.gov/about-nih/who-we-are/nihdirector/statements/statement-nih-funding-research-using-gene-editing-technologieshuman-embryos Accessed 15th March 2018. 
The NIH here suggests three reasons for opposition to germline interventions in the genome: there are safety issues that cannot be quantified; future persons born as a result of these technologies cannot consent to their use (since the technologies must, by necessity, be used before the people they give rise to exist); and there is no medically compelling case to use them.

Again, these reasons apply to interventions in all of the suggested mechanisms by which later generations come to resemble earlier ones, regardless of whether those mechanisms are described as forms of genetic germline inheritance, non-genetic germline inheritance, or non-germline inheritance. All of these mechanisms for inheritance affect members of future generations who are unable to offer consent. The need to ask whether there are compelling clinical rationales to intervene on these forms of inheritance is raised in all of these cases, too.

If we are dealing with genome-editing, the specific questions we need to ask include whether better-established forms of genetic screening-or entirely different interventions - might deliver similar outcomes in terms of disease transmission, but at lower risk. These sorts of considerations have been levelled against He Jankui's recent claim to have used gene editing to introduce a variant conferring immunity to HIV. That intervention seems reckless when there are other less risky ways of 
avoiding HIV transmission. ${ }^{37}$ Sales et al (2017) recently asked whether epigenetic mechanisms might explain the transmission across generations of metabolic diseases. They noted that 'Evidence that the isolated germ cell can mediate offspring disease was recently described by Huypens and collaborators, who utilized in vitro fertilization to demonstrate that germ cells harvested from mice exposed to nutritional factors (low-fat diet, normal diet, and high-fat diet...) are able to transmit metabolic phenotypes to offspring'. ${ }^{38}$ We might eventually determine with confidence that epigenetic inheritance is implicated in the transmission of metabolic disease in humans, raising the possibility of using some form of epigenome editing to influence disease transmission. It would then be essential to ask whether the same outcome could be achieved at lower risk via some more conventional intervention in diet, or perhaps using a screening technology. If what makes it wrong to intervene in the nuclear germline is 'a current lack of compelling medical applications', then the very same concerns will bar many other forms of intervention in other forms of inheritance.

${ }^{37}$ For an overview of the immediate reaction to He's November 2018 announcement see Mills, P. What He Said. 28 $8^{\text {th }}$ November 2018:

http://nuffieldbioethics.org/blog/what-he-said

38 Sales, V., A. Ferguson-Smith \& M-E. Patti. Epigenetic Mechanisms of Transmission of Metabolic Disease across Generations. Cell Metab 2017; 25 p. 561. 
An even more detailed ethical discussion can be found in the AAAS's document on what they call 'Human Inheritable Genetic Modifications'. They restrict the scope of their discussion to genes: ${ }^{39}$

IGM [Inheritable Genetic Modifications], as used in this report, refers to the technologies, techniques and interventions that are capable of modifying the set of genes that a subject has available to transmit to his or her offspring.

While narrowing the scope of discussion at this point in their document, they also give a very general account of the concerns raised by these technologies that seems to directly implicate many non-genetic ways of intervening in the processes of inheritance:

The kinds of interventions that fall within the scope of the definition of IGM are those that raise the following core issues:

- They are interventions that hold out the prospect of increasing our control over the specific hereditary traits of the next generation and beyond if they succeed;

- They are interventions that make inheritable changes in the genes of surviving offspring, rather than interventions that simply select among offspring on the basis of their naturally inherited genes;

39 Frankel \& Chapman op. cit. note 31. 
- They are interventions associated with scientific research, i.e., biomedical interventions, rather than social practices;

- They pose the risk of creating iatrogenic and other genetic harms.

There is an instability in the AAAS's treatment of these issues. Their list of the concern-raising characteristics of interventions that fall 'within the scope' of the definition of IGM implies that the term 'IGM' might reasonably stretch to cover a very wide range of interventions that introduce inheritable changes to offspring, whether mediated by genes or not. The worries triggered by genetic modification are also triggered in similar forms by epigenetic germline alterations, and by non-germline inherited alterations. In all of these cases, we raise the prospect of controlling the inherited traits of subsequent generations; we may aim to introduce changes in identified individuals, as opposed to selecting among several different offspring; wellintentioned medical intervention may inadvertently damage the health prospects of future generations; all of this may end up being done in the name of biomedical science; and it may thus be an iatrogenic form of harm.

If we return to the NIH's worries about genome editing, then we find that the ethical problems it raises for this technology apply not only to genome editing, but to nutritional advice designed to provoke epigenetic modifications to the germline, and even to urban planning, or changes to the organisation of schooling and specified educational curricula, that are meant to better the lot of future individuals. Here, too, we find interventions whose effects are uncertain, whose effects may also persist 
over several generations, and to which those affected by the changes-since they may not exist until after these structures have been put in place-cannot consent.

These remarks - which use germline epigenetic inheritance to draw parallels between nuclear genome editing, public health advice relating to nutrition, and urban planning-may seem flippant. They are not intended to be. We have good reason to ask to very same kinds of questions, whether we affect future people via their germ cells, their social and technical environments, or combinations of both. Urban planning, like genome editing and the provision of broad nutritional advice, has effects that are uncertain, persistent and hard to reverse. In all cases we should ask whether the interests of future people have been properly taken into account in our discussions, whether our uncertainties are properly reflected in a precautionary approach to action, whether these risky interventions are justified by genuine need, and whether we are recklessly introducing potentially harmful changes with longterm effects in blinkered service of a notion of technical progress.

\section{EPIGENETICS AND IDENTITY}

Even if non-genetic forms of inheritance raise many of the same ethical questions as interventions in the germline genome, it does not follow that there are no ethical features of germline genomic interventions that are distinctive. Moreover, a form of 'exceptionalism by degrees' might be justified, whereby the ethical problems raised by genomic interventions are far more acute versions of more familiar problems 
posed in other contexts of inheritance. ${ }^{40}$ The remaining sections consider two arguments that might establish alterations to the nuclear genome as deserving of special ethical oversight, hence which might justify the tendency to focus only on changes to nuclear genetic structures when we consider the rights and wrongs of germline interventions. The first concerns the allegedly special link between nuclear genes and 'identity'.

Sally Davies, the UK Chief Medical Officer, has suggested that changes to nuclear genes, unlike other interventions on the determinants of inheritance, have a special impact on traits that are relevant to an individual's identity. She commented on this matter as she tried to clarify the Department of Health's position on 'genetic modification', which it expressed in the context of its consultation on mitochondrial 'donation' technologies. ${ }^{41}$ The Department of Health noted that, 'There is no

${ }^{40}$ Lewens, T. Development Aid: On Ontogeny and Ethics. Stud Hist Philos Biol Biomed Sci 2002 33: 195-217.

${ }^{41}$ Department of Health. 2014. Mitochondrial Donation: Government Response to the Consultation on Draft Regulations to Permit the Use of New Treatment Techniques to Prevent the Transmission of a Serious Mitochondrial Disease from Mother to Child. Department of Health. The term 'mitochondrial donation' gives the impression that only mitochondria are transferred from the donated eggs of a healthy woman to the intended mother. In fact, the donor contributes all cellular structures with the sole exception of nuclear material that comes from the intended mother. See Lewens, T. 2015. Biological Foundations of Bioethics. Oxford: Oxford University 
universally agreed definition of 'genetic modification' in humans.... The working definition that we have adopted is that genetic modification involves the germline modification of nuclear DNA (in the chromosomes) that can be passed on to future generations'. ${ }^{42}$

Davies tried to justify the Department of Health's reasoning when she was questioned by the House of Commons Science and Technology Committee:

...we needed to make the distinction between nuclear DNA, which makes us who we are and how we are - our personalities, heights, weights and whether or not we get baldness - and the 37 genes in the mitochondria which are about energy for the cell, and which we describe as the power pack. That was why we adopted that working definition. ${ }^{43}$

Davies suggests here that it is the nuclear DNA that 'make us who we are'. The implication is that mitochondrial genes, and, by extension, the molecules potentially involved in epigenetic inheritance, do not have this identity-determining role.

Press; Lewens, T. The Division of Advisory Labour: The Case of 'Mitochondrial Donation’ Eur J Philos Sci 2019; 9: https://doi.org/10.1007/s13194-018-0235-3 42 lbid.

${ }^{43}$ Sally Davies, quoted in Scott \& Wilkinson op. cit. note 29. 
The experience of disease can be a remarkably strong element of an individual's self-conception, hence of 'identity' in that important respect. To the extent that nonnuclear DNA affects disease phenotypes, it follows that we should also credit elements of mitochondrial genomes, and also inherited epigenetic structures, with a role in determining identity.

A more charitable reading of what Davies intended by her remarks is that she was expressing concern with the potential for changes in the nuclear genome to exert very fine-grained control over positively valued cognitive, emotional, behavioural or physiological traits, as opposed to making large-scale differences to the presence or absence of disease traits.

In response to this concern, we should note that it will tend to licence some germline interventions to the nuclear genome, so long as they are also of a kind that go no further than affecting the presence or absence of disease. There are disorders of mitochondrial function that arise not from disorders of the mitochondrial genome, but rather from disorders in nuclear genes that influence mitochondrial function. ${ }^{44}$ The clinical conditions of people with nuclear, compared with mitochondrial, genetic abnormalities can be very similar. It is hard to see how one might come to the verdict that inheritable interventions on the mitochondrial genome are unproblematic by virtue of having nothing to do with 'identity', unless we also agree that the necessary

${ }^{44}$ NCOB. 2012. Novel Techniques for the Prevention of Mitochondrial DNA Disorders: An Ethical Review. London: Nuffield Council on Bioethics: 1: 11. 
inheritable interventions to the nuclear genome, when they seek to remove precisely the same kinds of inherited clinical symptoms, are also unproblematic in this respect.

It is also an open question whether, and how often, intervening on nuclear genes will truly enable more fine-grained control over valued phenotypes. To the extent that phenomena of epistasis (in particular, the contingency of effects of gene substitutions on genetic backgrounds that can vary from individual to individual) turn out to be pervasive, as well as forms of pleiotropy (i.e. a gene having multiple effects on different phenotypes), then intervention on nuclear genes may be unwise as a method of trying to control phenotypic outcomes, because of the unpredictable effects of gene alterations on traits other than the target phenotypes of interest. ${ }^{45}$ Further, to the extent that the phenotypic effects of nuclear genes are very small in magnitude, then if we are aiming to control valued cognitive, emotional or physiological traits, then we may simply find that direct interventions on other developmental processes - related to such mundane things as nutrition, training or education - will be far more efficient. ${ }^{46}$

\section{THE ‘REVERSIBILITY’ OF EPIGENETIC INTERVENTIONS}

The Nuffield Council on Bioethics noted that opposition to genome editing has been especially intense 'where scope for unforeseen consequences is considered to be

\footnotetext{
${ }^{45}$ See NCOB op. cit. note 29: 4: 43.

46 Lewens, T. op. cit. note 39.
} 
great or editing is regarded as irreversible'. ${ }^{47} \mid$ argue in this penultimate section that the notion of 'irreversibility' is unlikely to be able to ground a general ethical asymmetry between genomic, and epigenetic inheritance.

Bohacek \& Mansuy, in their discussion of the epigenetic inheritance of behavioural phenotypes, reported that while foetal exposure to alcohol in rats could increase their sensitivity to stress over two or three generations, 'Some of the inherited effects can be corrected by environmental enrichment in adolescent rat offspring, suggesting reversibility of symptoms' ${ }^{48}$ Many effects of germ-line genetic alterations are 'reversible' in just the same sense: even when genes are inherited across numerous generations, their phenotypic effects will often be modifiable by altering the environment of development.

Moreover, if genome engineering becomes a very powerful tool for intervening on nuclear germline DNA sequence, then in theory the very same technology that allows genomic changes in one direction will allow the same changes to be undone in the opposite direction. This does not mean our efforts at reversal will be successful. It is also unlikely that we will be able to track down and intervene on all later individuals affected by an inherited genomic alteration. But this does not establish a sharp difference between genomic and epigenetic interventions to inheritance systems. If we start to intervene on non-genetic inheritance structures,

\footnotetext{
${ }^{47}$ NCOB op. cit. note 29.

${ }^{48}$ Bohacek \& Mansuy, op. cit. note 6, p. 642.
} 
we may also find it hard to control all of the effects of our actions. This is especially obvious when we think of modifications to the social and cultural determinants of inheritance in humans. If we institute new ways for designing long-lasting social housing projects, or if we issue sweeping new forms of public health advice that turn out to affect germline-inherited epigenetic structures, then it is wishful thinking to assume the effects of our actions on future generations will be easy to undo if we realise we have made mistakes.

These arguments leave open the possibility that there are important differences of degree between ethical features of nuclear genomic intervention, and interventions in other inheritance processes. Only further research will confirm whether, as many suspect, genomic interventions are more stable over time than other interventions. We have seen widespread agreement that many epigenetic states, such as methylation patterns, are 'reset' during the processes of reproduction, with the result that there is a significant difference between DNA sequence-based inheritance and epigenetic inheritance with respect to the number of generations for which modifications can be expected to be stable. We have also seen, however, that this appears not to be the case for all epigenetic states, and that there are live research question about the extent to which some forms of epigenetic inheritance are transgenerationally stable in mammals.

There is an important scientific distinction to be drawn, as we have seen, between intergenerational and transgenerational effects. Moreover, the jury is still out on the transgenerational nature of epigenetic inheritance in humans. Even so, it is unclear 
how the intergenerational/transgenerational distinction could allow us to establish a very sharp ethical line between interventions to germline DNA sequence and interventions that might affect other inheritable germline structures. Suppose our ethical concerns with germline genomic interventions rest on the worry that overzealous meddling will adversely affect multiple future generations. This would hardly show that, since most interventions affecting epigenetic inheritance will have effects that persist for only two or three generations, we should be unconcerned with them. We need to pay due ethical attention to a motley assortment of forms of inheritance mediated by everything from the built environment to histones and small RNAs, via social behaviour and the constituents of seminal fluid. Some of these do, and some do not, modify the germline, but there are indications that all may have impacts on the development of future generations.

\section{CONCLUSION}

In scientific contexts, the notion of a germline modification tends to receive a comparatively strict definition. It is not simply any intervention that is likely to be propagated over several generations. It is an intervention that is likely to be propagated over anything from two to an indefinite number of generations (depending on whether it is inter-generationally, or trans-generationally stable), and which acts via a modification to germ cells.

We have seen evidence that inheritable germline modifications, in this strict scientific sense of the term, do not uniquely pick out interventions to nuclear DNA sequence. 
Unfortunately, bioethical discussion often overlooks this. We have also seen that the very concerns that have triggered caution over modifications to germline nuclear DNA sequence-about the potential for poorly-understood, irreversible, multigenerational change; about consent; about the clinical rationale for using these new forms technical interventions - do not apply to germline modifications alone. There is increasing evidence for a class of inherited modifications that do not operate via modifications to the germline at all, and which trigger precisely the same set of ethical concerns. The result is that scientific work- when it operates in tandem with ethical reflection-is teaching us that there is no special sin of germline intervention.

It is important to stress the modesty of this result. It reminds us to ensure that regulation is not blind to the possibility of morally troubling interventions in nongenomic structures of inheritance. This does not mean that germline interventions raise no significant ethical problems. What is more, we might worry that a combination of (i) widespread enthusiasm for the promise of high-tech interventions, (ii) the comparative ease of using CRISPR-Cas9 to alter the genome, and (iii) the potential for such early interventions in developmental processes to do systemic damage to a growing child, together make the genome an especially likely locus for misguided efforts to improve the lot of future generations. As a result, we may have reasons to be more vigilant when it comes to genome editing compared with other ways of influencing inheritance processes. But reflection on the cases of nutritional advice and urban planning reminds us that any rigid stance against intervention in the processes of inheritance is absurd, since such interventions are inevitable and 
pervasive. The true significance of taking a humble attitude to our influence over the inheritance of future generations extends well beyond the germline.

\section{Conflict of Interest}

I have no conflicting interests

\section{Acknowledgements}

I am grateful to Anne Ferguson-Smith for some very early advice about this project, to Andy Greenfield for comments on a draft version, and to the John Templeton Foundation for funding. Earlier versions of this paper were given in Strasbourg, the EMBL Heidelberg, and the University of Cambridge, and I would like to thank audience members-including Thomas Pradeu, Lucy Laplane, Halldor Steffanson, Raphael Scholl, Stephen John, Jacob Stegenga, Joseph Wu and Anna Alexandrova-who were present at those presentations. I am especially grateful to two referees from this journal for their remarkably helpful reports. 